\title{
Full Color Generation Using Silver Tandem Nanodisks
}

\author{
Hao Wang, ${ }^{\dagger,, \S \odot ~ X i a o l o n g ~ W a n g, ~}{ }^{\dagger}$ Chen Yan, ${ }^{\dagger}$ Hua Zhao, $^{\ddagger, \S}$ Jingwen Zhang, $^{*, \ddagger}$, Christian Santschi, $^{\dagger}$ \\ and Olivier J. F. Martin*, ${ }^{*}$ (i) \\ ${ }^{\dagger}$ Nanophotonics and Metrology Laboratory (NAM), Swiss Federal Institute of Technology Lausanne (EPFL), 1015 Lausanne, \\ Switzerland \\ ${ }^{\ddagger}$ Institute of Modern Optics, Department of Physics and ${ }^{\S}$ Key Laboratory of Micro-Optics and Photonics Technology of Heilongjiang \\ Province, Harbin Institute of Technology, Harbin 150001, China
}

\section{Supporting Information}

ABSTRACT: Plasmonic effects associated with metallic nanostructures have been widely studied for color generation. It became apparent that highly saturated and bright colors are hard to obtain, and very small nanostructures need to be fabricated. To address this issue, in this study, we employ metal-insulator-metal sandwich nanodisks that support enhanced in-phase electric dipole modes, which are blueshifted with respect to a single metal disk. The blue shift enables the generation of short wavelength colors with larger nanostructures. The radiation modes hybridize with the Wood's anomaly in periodic structures, creating narrow and high-resonance peaks in the reflection and deep valleys in the transmission spectra, thus producing vivid complementary colors in both cases. Full colors can be achieved by tuning the radius of the nanodisks and the periodicity of the arrays. Good agreement between simulations and experiments is demonstrated and analyzed in CIE1931, sRGB, and HSV color spaces. The presented method has potential for applications in imaging, data storage, ultrafine displays, and plasmon-based biosensors.

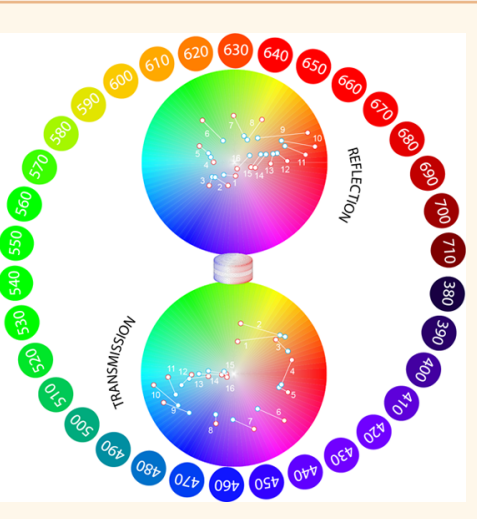

KEYWORDS: metasurface, tandem nanodisks, metal-insulator-metal nanodisks, color, localized surface plasmon resonance, reflection and transmission

$\mathrm{M}$ anipulating the interaction between light and matter at different length scales has been explored and applied since the ancient ages in human history, during which colors generated by nanoscale metallic objects have been used to create exquisite decorations and patterns, such as nanoparticles (NPs) in stained glasses and the wellknown dichroic Lycurgus Cup. ${ }^{1}$ These effects relied on localized surface plasmon resonances (LSPRs) of NPs and their associated strong optical response that can be tuned by changing the NPs' size, shape, arrangement, or material. Recently, with the aim toward higher resolution, higher fidelity, color tunability, and ultrathin devices for imaging and display technologies, plasmonic nanostructures have received a burgeoning amount of interest driven by their ability to generate a wide range of colors by tuning the plasmon resonance. ${ }^{2-4}$ Using metallic nanoantenna arrays with aluminum $(\mathrm{Al}),{ }^{5-10}$ silver $(\mathrm{Ag}),{ }^{11-13}$ and gold $(\mathrm{Au}),{ }^{14,15}$ different colors have been realized by varying the geometry and the period of the antennas. ${ }^{2}$ Nanoholes in a metal film have also been intensively studied because of the enhanced transmission at specific frequencies associated with the excitation of surface plasmon polaritons (SPPs) at the metal surface in the vicinity of apertures. ${ }^{15-20}$ The central excitation frequency of SPPs can be tuned by varying the apertures' size, shape, and distribution. In addition, plasmonic hybridization induced by disk-hole coupling $^{21-26}$ results in a low-energy (bonding) mode and a high-energy (antibonding) mode. Changing the physical structure of the disk and aperture is an efficient method to shift the bonding mode to make color filters. Besides, gap plasmon-based reflective color filters also provide a reliable way to encode information into subwavelength color printing. ${ }^{27}$ The possibility to excite plasmon modes at two different wavelengths, that is, different colors, in orthogonal directions enables color selection between them with an appropriate polarization. $^{15,28-30}$

However, the spectra obtained with plasmonic structures exhibit a relatively large full width at half-maximum (fwhm). and the peak heights and valley depths are relatively small, 6,10,19-22,25-29 which reduce the saturation and brightness performances. To achieve purer colors, a Febry-Pérot cavity with multilayer films, ${ }^{31-40}$ guided mode resonances, ${ }^{41}$ or gratings ${ }^{42-50}$ have been employed to realize high saturation

Received: December 17, 2016

Accepted: March 20, 2017

Published: March 20, 2017 
colors and high brightness, although they are not suitable for ultrasmall pixel design when compared to plasmonic nanoantenna arrrays. Dielectric metasurfaces with high refractive index, such as silicon $(\mathrm{Si}),^{51-53}$ have also been explored recently, although they also suffer from limited saturation and brightness. In addition, most of the previous reports on plasmonic colors have mainly focused on the hue performances and lack an analysis of saturation and brightness.

To tackle the challenge of highly saturated and bright color generation with plasmonic structures and maintain the pixel design functionality, we use tandem nanodisk arrays to realize vivid red-green-blue (RGB) colors in reflection and cyanmagenta-yellow (CMY) colors in transmission. The main principle used here is the hybridization between Wood's anomaly and the enhanced in-phase electric dipole mode of the nanodisks pair, which produces a high narrow peak in reflection and a deep valley in transmission when choosing the appropriate radius and period. In addition, this method enables the fabrication of short wavelength colors with relatively large nanostructures. Simulations and experiments agree very well and are also compared with the Commission Internationale de l'Eclairage (CIE), ${ }^{54}$ standard RGB (sRGB), and hue saturation value/brightness (HSV/HSB) color spaces. The structure discussed in this paper can be applied for encryption, holograms, high-density optical data storage, biosensors, and security applications.

\section{RESULTS AND DISCUSSION}

Figure 1a shows the schematic drawing of a tandem nanodisk square array with period $P$ and radius $R$ on a silica $\left(\mathrm{SiO}_{2}\right)$ glass substrate. The thicknesses of the $\mathrm{Ag}$ and aluminum oxide $\left(\mathrm{Al}_{2} \mathrm{O}_{3}\right)$ layers are $H=30 \mathrm{~nm}$ and $h=20 \mathrm{~nm}$, respectively. These two parameters have been first optimized to obtain the highest reflection peak at $P=380 \mathrm{~nm}$ and $R=80 \mathrm{~nm}$ (Figure $S 1$ in Supporting Information). The thicknesses are maintained the same for all colors in the following study, thus enabling one to print a broad variety of colors on the same substrate. The sample is prepared with the procedures described in the Methods section and Figure S2 in Supporting Information. A $52^{\circ}$ side view of the scanning electron microscopic (SEM) image of the fabricated nanostructures is shown in Figure $1 \mathrm{~b}$.

For a single tandem structure, the scattering cross section spectrum has a broad resonance at high frequency $\omega_{+}$and a narrow one at low frequency $\omega_{-}$, as shown in Figure 1c. The optical response of this metal-insulator-metal (MIM) sandwich structure can be qualitatively understood by considering the two metal nanodisks as coupled point dipoles and described as two oblate spheroids with polarizability $\alpha^{11,55}$

$$
\alpha_{i}(\omega)=\frac{R_{i}{ }^{2} r_{i}}{3 L_{i}} \frac{\omega_{0 i}^{2}}{\omega_{0 i}^{2}-\omega^{2}-i \omega \nu_{\mathrm{c}}}
$$

where $i=1,2 ; R_{i}$ and $r_{i}$ are the long and short radii of spheroid $i$, respectively; $L_{i}$ is the geometrical factor of a spheroid; $\omega_{0 i}$ and $\omega$ are the localized surface plasmon resonance frequency and light frequency in vacuum, respectively; and $\nu_{\mathrm{c}}$ is the collision rate for the electrons in the spheroid metal. When the sandwich structure is illuminated from the top, the two polarizabilities hybridize with each other, leading to the two hybridized plasmon frequencies ${ }^{55}$
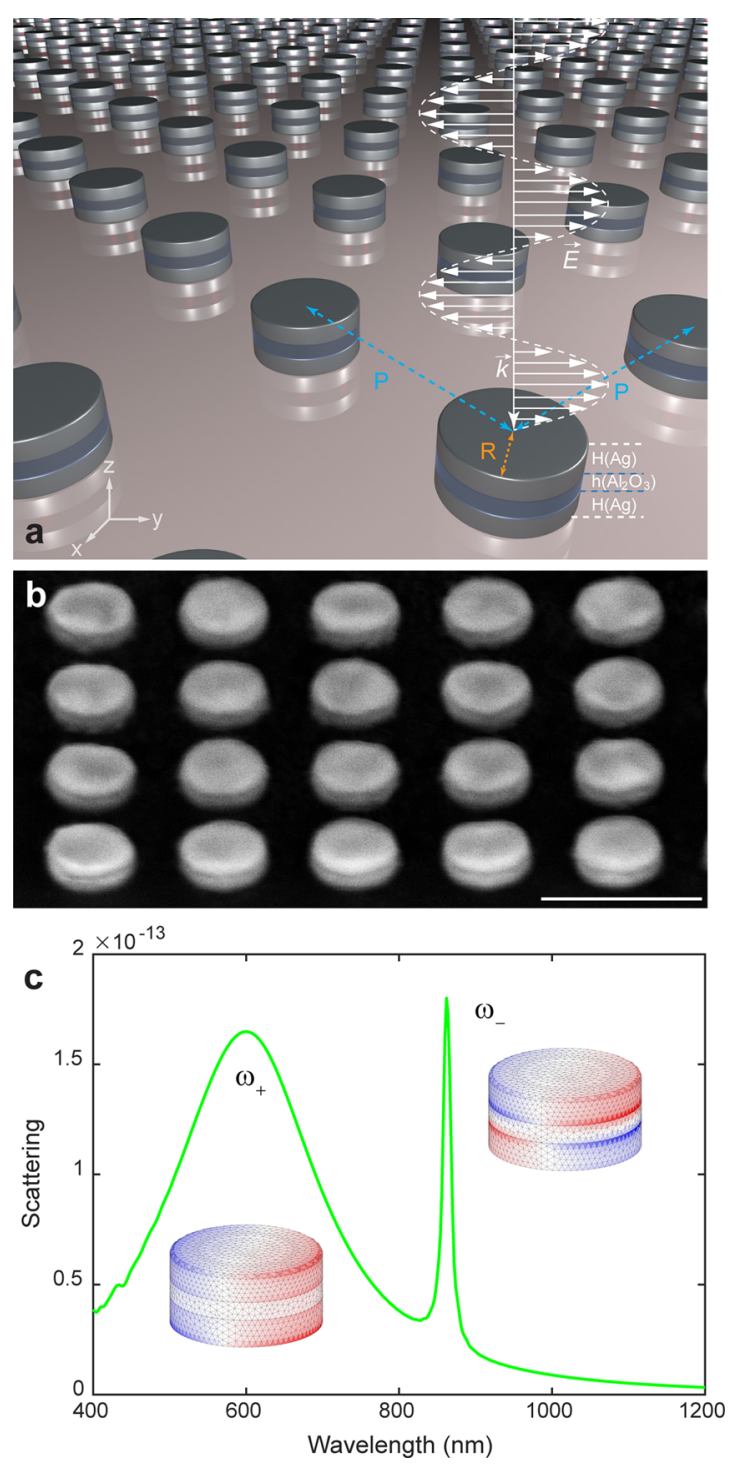

Figure 1. (a) Schematic drawing of the tandem nanodisk square array with period $P$, radius $R$, and thicknesses of the $\mathrm{Ag}$ layers $H$ and of the $\mathrm{Al}_{2} \mathrm{O}_{3}$ layer $h$. The structure is illuminated at normal incidence with $y$-polarized white light. (b) SEM image of the fabricated sample in $52^{\circ}$ side view; scale bar: $500 \mathrm{~nm}$. (c) Calculated scattering cross section spectrum for a single in-tandem structure. The insets represent the corresponding charge distributions for the in-phase and the out-of-phase modes.

$$
\begin{aligned}
\omega_{ \pm}^{2}= & \frac{1}{2}\left(\omega_{01}^{2}+\omega_{02}^{2}\right) \\
& {\left[1 \pm \sqrt{1-\frac{2\left(1-R_{1}^{2} R_{2}^{2} r_{1} r_{2} /\left(L_{1} L_{2} d^{6}\right)\right) \omega_{01}^{2} \omega_{02}^{2}}{\left(\omega_{01}^{2}+\omega_{02}^{2}\right)^{2}}}\right] }
\end{aligned}
$$

where $d$ is the distance between the centers of the two oblate spheroids. The two resonant modes are identified as an inphase $\left(\omega_{+}\right.$, symmetric $)$mode at higher frequency and an out-ofphase $\left(\omega_{-}\right.$, asymmetric) mode at lower frequency. ${ }^{56}$ The corresponding charge distributions can be seen from the insets in Figure 1c. Other approaches such as the homogeneous uniaxial layer approach, the dynamic Yamaguchi approach, or the island film theory can also be applied to get insights into this structure. ${ }^{57}$ The low-energy asymmetric mode produces a 

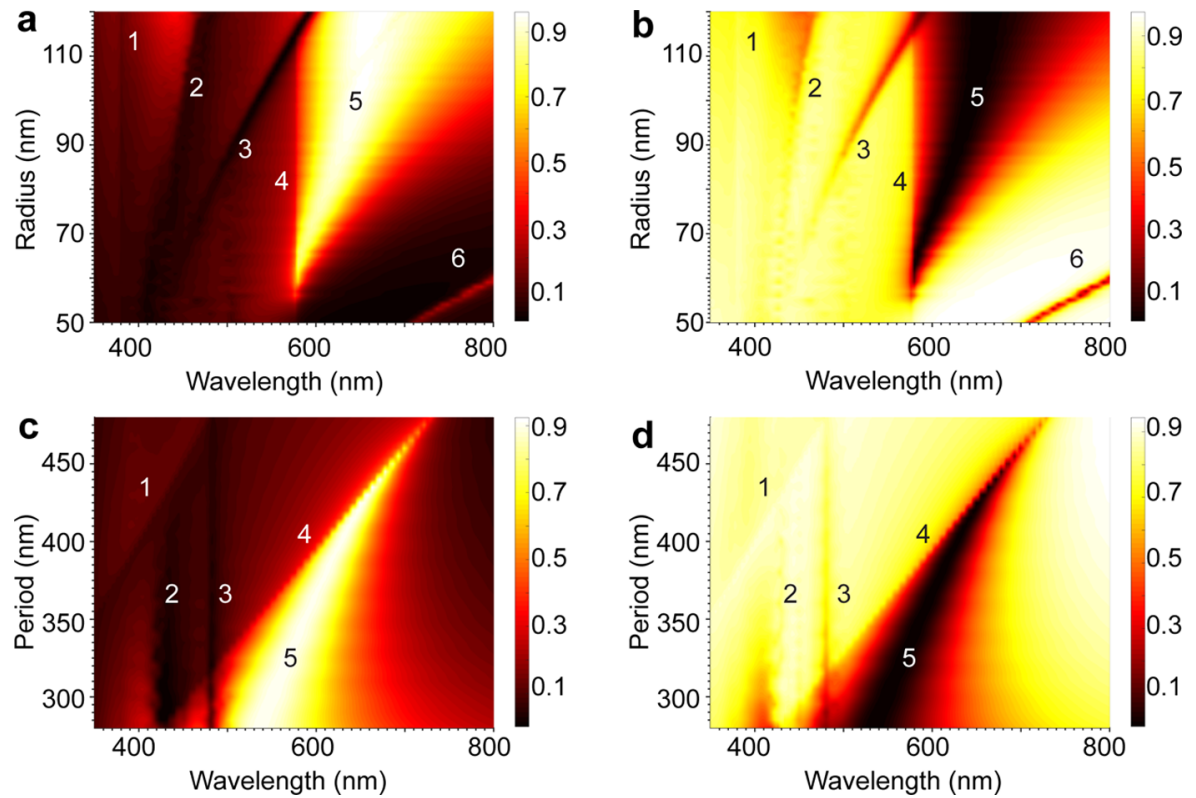

Figure 2. (a) Reflection and (b) transmission for nanodisk arrays with a period of $380 \mathrm{~nm}$ and radii ranging from 50 to $120 \mathrm{~nm}$. (c) Reflection and (d) transmission for nanodisk arrays with a radius of $80 \mathrm{~nm}$ and periods ranging from 280 to $480 \mathrm{~nm}$.
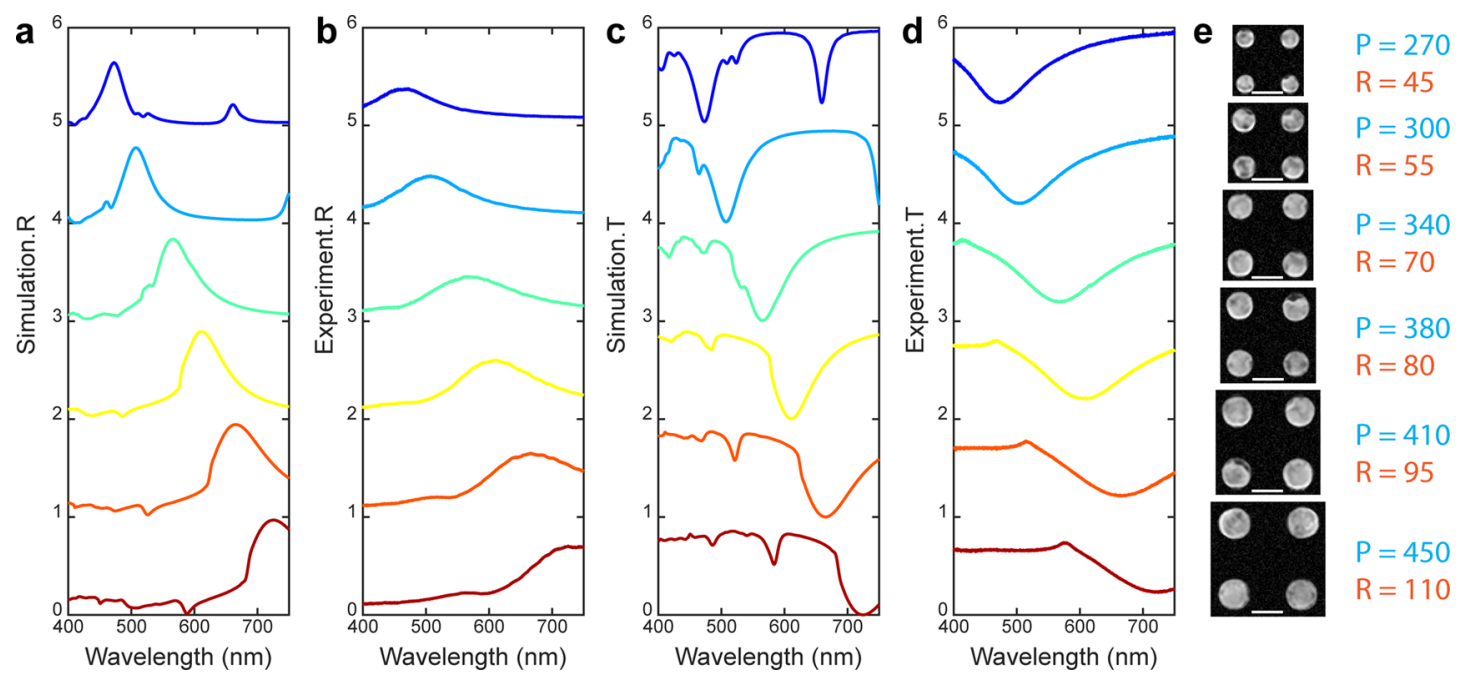

Figure 3. (a) Simulated and (b) experimental reflection spectra. (c) Simulated and (d) experimental transmission spectra. (e) Corresponding SEM images with period and radius values indicated on the right. Scale bar: $200 \mathrm{~nm}$.

virtual current loop due to retardation effects; therefore, a magnetic moment is generated and the magnetic field is enhanced between the two metal disks. This has been used in surface-enhanced Raman scattering (SERS), ${ }^{58-60}$ sensors, ${ }^{61,62}$ high Purcell factors, ${ }^{63}$ negative refractive indexes, ${ }^{64}$ and so on. Particularly, trapezoidal sandwich structures can further enhance the magnetic field and exhibit Fano resonances. ${ }^{65-69}$ The sharpness of the resonance can be measured by the quality factor ( $Q$ factor, which is proportional to the resonance frequency divided by the fwhm); the $Q$ factor of the out-ofphase mode is higher than that of the in-phase mode, as seen from Figure 1c. However, most of the energy radiation is caused by the latter mode, in addition, whose resonance peak is enhanced and blue-shifted when compared with the single nanodisk, ${ }^{61,70,71}$ which is beneficial for full color generation because larger structures are easier to fabricate (see the quantitative comparison of resonances between the sandwich structure and single nanodisks in Figure S3 in the Supporting
Information). ${ }^{72}$ To take advantage of this, the whole sandwich structure is chosen to have the same radii between the top and bottom disks to minimize the magnetic mode and enhance the electric mode.

In the simulations with finite-difference time domain (FDTD) and a surface integral equation (SIE) (see Methods), ${ }^{73-75}$ the light with $y$-polarization is at normal incidence on the in-tandem structure array, as shown in Figure 1a. Figure $2 \mathrm{a}, \mathrm{b}$ shows the reflection and transmission of the nanodisk array with fixed period $P=380 \mathrm{~nm}$ and varying radius $R$ from 50 to $120 \mathrm{~nm}$ (see the dependence of reflection and transmission on the incident angle for TE and TM polarizations in Figure S4 in the Supporting Information). The vertical features labeled with 1 and 4 are caused by Wood's anomalies in air and on the substrate, respectively; those labeled with 2 and 3 correspond to the high-order modes ${ }^{76-78}$ of the sandwich structure (see charge distribution in Figure S5 in the Supporting Information), which has also been confirmed by 

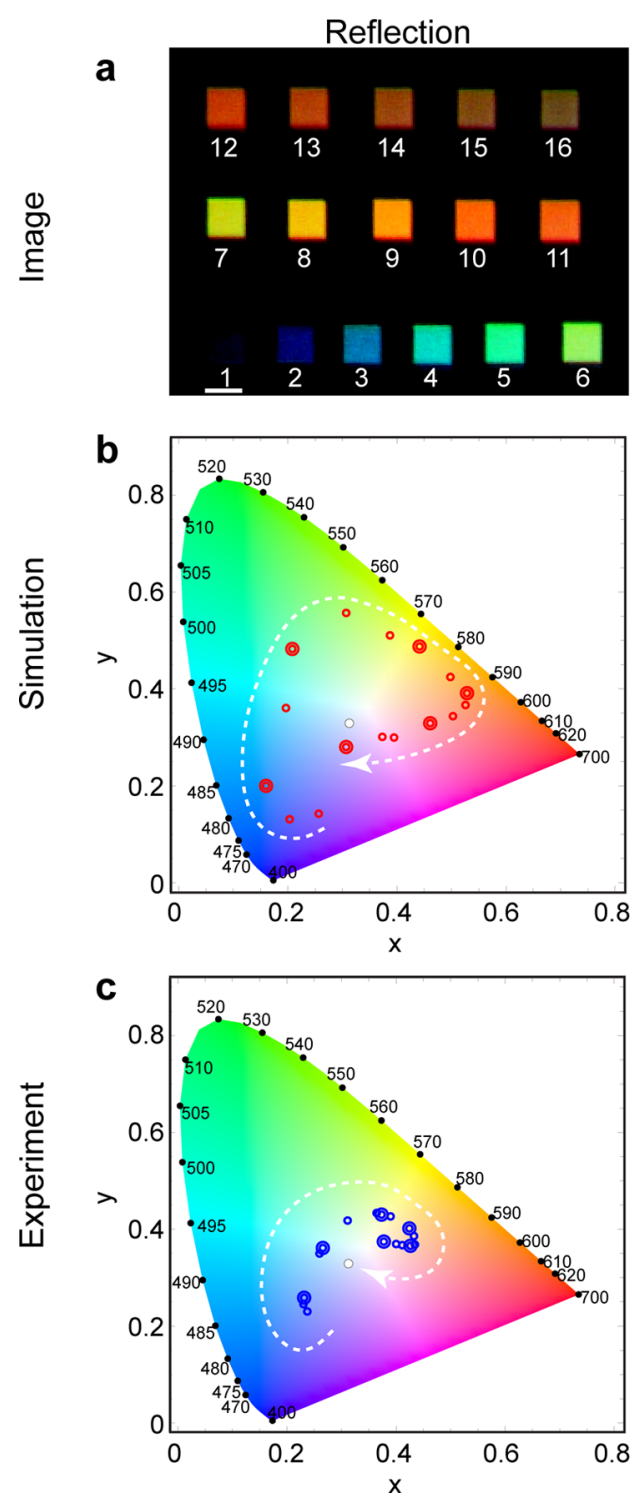

d Transmission
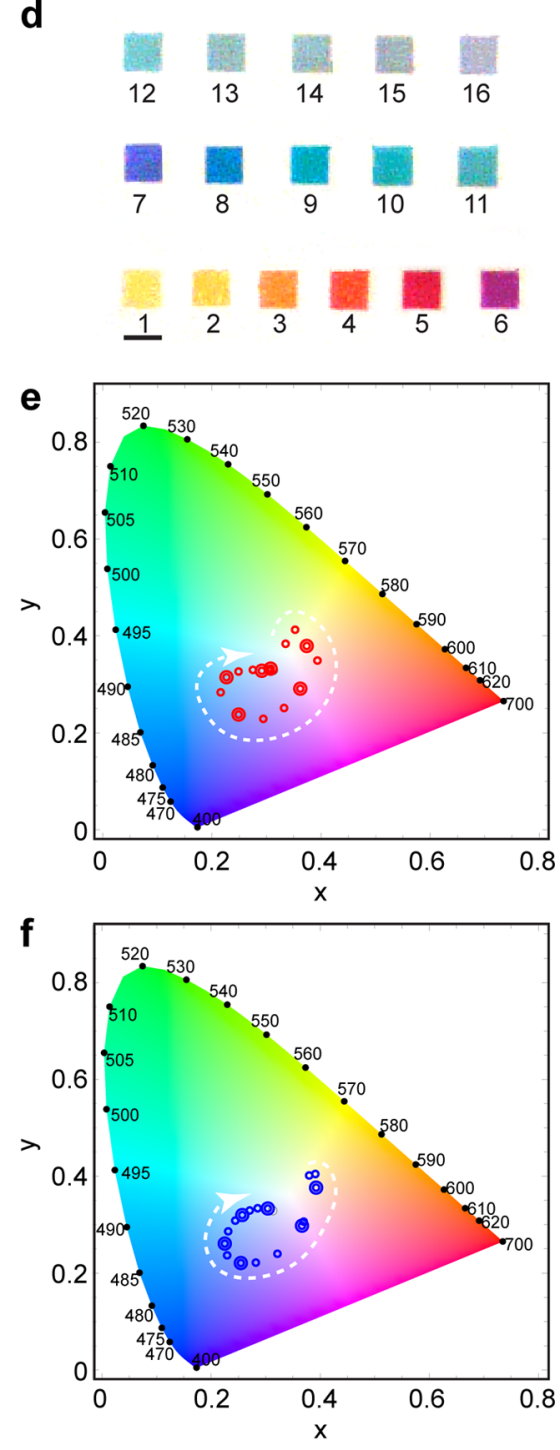

Figure 4. Reflection (left column) and transmission (right column) colors for tandem nanodisk arrays with different radii and periods. Measured (a) reflection (from dark blue to red) and (d) transmission (from yellow to cyan) image colors for 16 samples. Scale bar: $40 \mu \mathrm{m}$. $(b, e)$ Simulated colors and (c,f) experimental colors. The six colors corresponding to Figure 3 are marked with double rings. The white dashed lines with the arrow represent the evolution trend for the colors when the radius and period are increased.

varying the value of the period $P$ from 280 to $480 \mathrm{~nm}$ at a fixed radius $R=80 \mathrm{~nm}$; features 2 and 3 do not shift with the period (Figure $2 \mathrm{c}, \mathrm{d}$ ). The mode labeled with 6 corresponds to the outof-phase mode (see magnetic field in Figure S5 in the Supporting Information). The main resonance (labeled with 5 ) is the hybridization mode between the in-phase mode and Wood's anomaly (4), which generates a relative narrow resonance when compared with that of a single structure (compare with Figure 1c). When the radius and period are reduced, the main peak from the hybrid mode undergoes a blue shift, thus generating a different color (Figure S6 in the Supporting Information). By varying both $P$ and $R$ in the simulation, we can obtain their optimized combination for one color, at which a good balance between the fwhm and the magnitude of the peak/depth of the valley can be reached. This way, all of the colors can be obtained by different parameter combinations of the period and the radius. Here, we inspect 16 different samples with periods $P$ ranging from 250 to $450 \mathrm{~nm}$ and radii $R$ from 35 to $110 \mathrm{~nm}$ (see the parameter combinations in Table $\mathrm{S} 1$ in the Supporting Information). Figure 3 shows the simulated and experimental reflection and transmission spectra for six typical colors with the corresponding SEM images and parameters listed on right (see full experimental reflection and transmission spectra for 16 samples in Figures S7 and S8 in the Supporting Information). The experimental results (Figure $3 \mathrm{~b}, \mathrm{~d}$ ) are in good agreement with the simulations (Figure 3a,c). Peaks and valleys induced either by the out-of-phase mode or by the high-order modes are not observed in the experiment. One reason is because of the inhomogeneous broadening caused by the defects in fabricated nanostructures, as illustrated in Figure $3 \mathrm{e}$; another one is that they are perturbed by the surface fluctuations of the deposited $\mathrm{Al}_{2} \mathrm{O}_{3}$ film in the sandwich structure. Inhomogeneous broadening also widens the main resonance from the hybrid mode, whereas the fwhm still remains narrow. Although the peak heights and dip depths are slightly reduced, we measure extremely good performances with $70 \%$ reflection and $18 \%$ transmission efficiencies (97 and 1\% for the simulations). 
Reflection

a

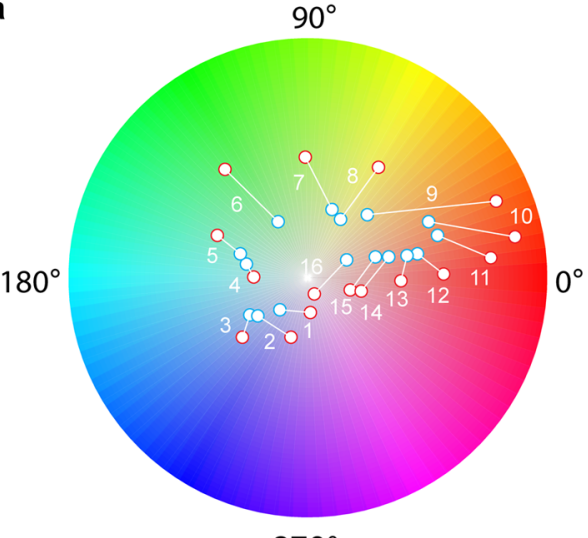

$270^{\circ}$
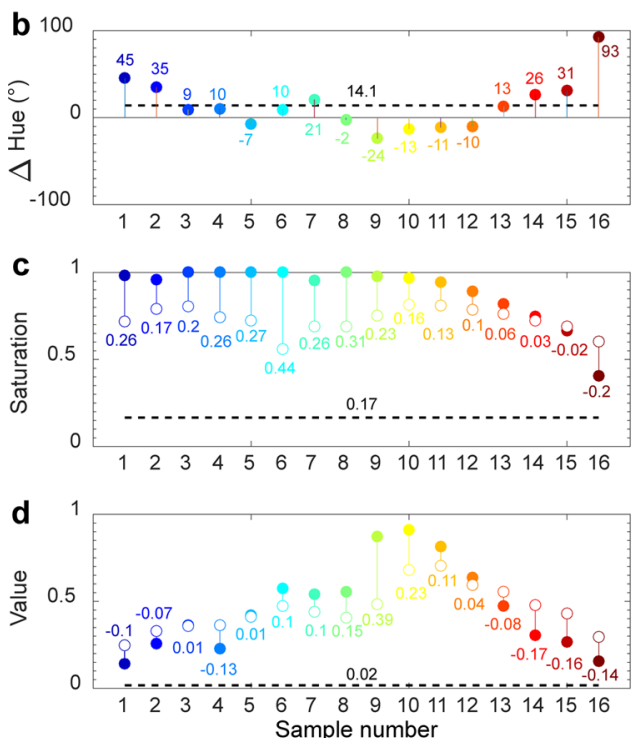

Transmission

$\mathbf{e}$
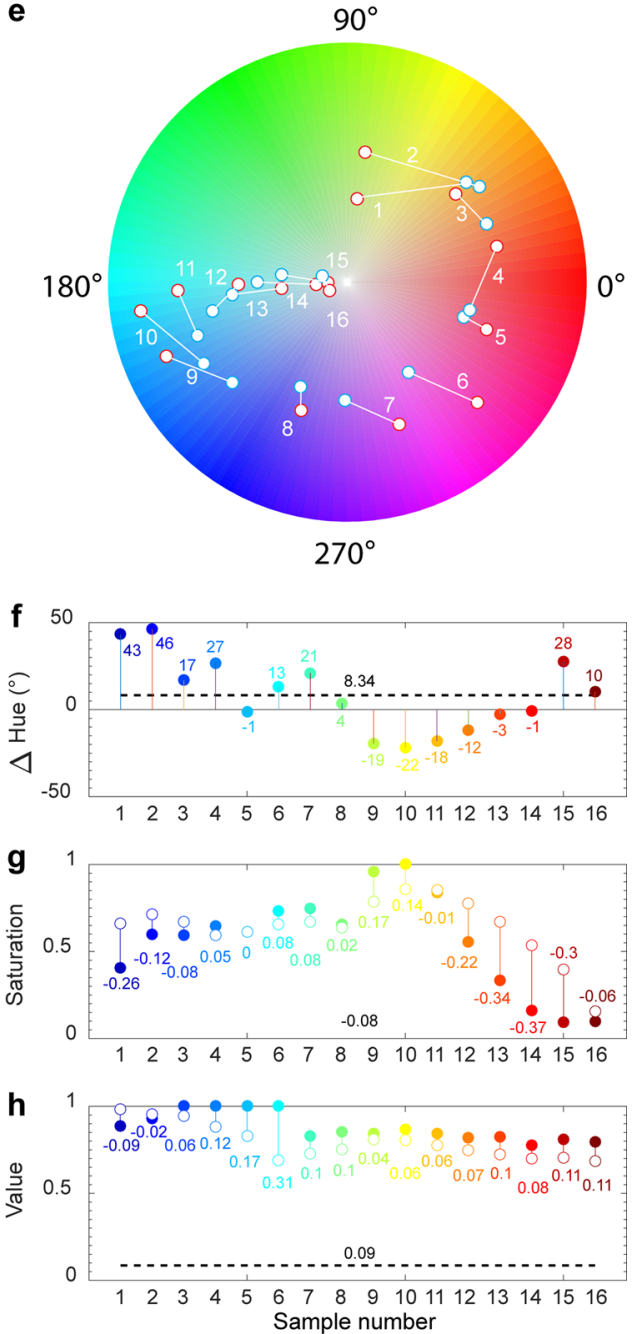

Figure 5. Reflection (left) and transmission (right) colors in HSV color space. (a,e) Top views of the HSV color cones with hue and saturation information on simulated (red hollow circles) and experimental colors (blue hollow circles). (b,f) Color differences (simulation experiment) for hue $-\Delta^{\text {Hue }}$. (c,g) Saturation and (d,h) value (brightness) for simulations (solid circles) and experiments (hollow circles), respectively. The labels correspond to the differences. The black dashed lines and numbers represent the average differences between simulation and experiment in the corresponding diagrams.

The experimental vivid color images for reflection and transmission are shown in Figure $4 \mathrm{a}, \mathrm{d}$, respectively (see simulated colors in Figure S9 in the Supporting Information). As seen from these pictures, with increasing radius and period, the reflection color varies from dark blue (at the bottom-left corner) to red (at the top-right corner); synchronously, the transmission color changes from yellow to cyan. To compare the color performances between the experimental results and the simulations, we first calculate the tri-stimulus values by integrating them over the visible range of wavelengths with the following equation ${ }^{54}$

$$
M=k \int_{360}^{780} E(\lambda) P(\lambda) m(\lambda) \mathrm{d} \lambda
$$

where $M=X, Y, Z$ and the corresponding $m$ is $x, y, z$, respectively. ${ }^{54}$ In eq $3, k$ is a normalizing factor; $E(\lambda)$ is the relative spectral power distribution of the illuminant; $P(\lambda)$ is the spectral reflectance/transmittance of the surface, that is, the simulated and experimental spectra; $m(\lambda)$ is the color-matching function. Here, color-matching functions with CIE1931 $2^{\circ}$ standard observer under illumination D65 from the American Society for Testing and Materials E308-01 (ASTM, 2001) (79 $^{79}$ employed, and the data are interpolated with a resolution of 1 $\mathrm{nm}$ from the original data at $10 \mathrm{~nm}$ interval (Figure S10 in the Supporting Information). The chromaticity coordinates can then be computed from the tri-stimulus values by

$$
m=M /(X+Y+Z)
$$

In the CIE1931 chromaticity space, it is convenient to visualize the color trends (represented by dashed white lines with arrow in Figure 4)-reflection colors in blue-green-red sequence and transmission in complementary colors yellow-magentacyan sequence, with increasing radius and period. The experimental colors (marked in blue) fit well with the simulation ones (marked in red). For comparison, the corresponding colors of the simulated and experimental spectra in Figure 3 are marked with double rings. In contrast to Figure $4 \mathrm{~b}$, the experimental reflection colors in Figure $4 \mathrm{c}$ converge toward the white point (marked with the white spot, illumination D65), which is due to the broadened resonance 
peaks and the elevated intensity out of resonance, whereas the experimental transmission case in Figure $4 \mathrm{f}$ is quite similar to the simulation in Figure 4e. When the spectra are multiplied with the Gaussian-type color-matching functions, ${ }^{80}$ we can easily draw the conclusion that in our structure the reflection colors are more sensitive than the transmission ones. The colors are additionally compared in the sRGB and CIEXYZ color spaces considering the luminance $Y$ (Figure S11 in the Supporting Information). We can see that, by using sandwich structures, a wide range of colors can be generated in the sRGB space. In addition, due to the value of $Y$ being equivalent to luminance expressed in units of $\mathrm{cd} / \mathrm{m}^{2}$ for the 1931 standard observer, uniform luminance for these colors can be realized by adjusting the power distribution in the illuminant spectrum.

To further analyze these colors numerically, we convert them into the HSV color space by color matrix transformation (see the HSV color cones in Figure $\mathrm{S} 12$ in the Supporting Information). Figure 5a,e shows the top views for the HSV color cones for reflection and transmission, respectively. The experimental results (blue hollow circles) are linked with their simulated counterparts (red hollow circles) and labeled with sequence numbers according to Figure 4. The differences (simulation - experiment) of hue $\left(\Delta^{\text {Hue }}\right)$ in Figure $5 b, f$ are mostly in the small range between -24 and $+45^{\circ}$ for reflection and between -22 and $+46^{\circ}$ for transmission. The large deviation from simulation, $93^{\circ}$ for the 16 th sample in the reflection, can be understood by the rather small amount of red color-matching function around $700 \mathrm{~nm}$, which means that the real color here is very sensitive to the line shape of the spectrum, while people's eyes are relatively insensitive in this range. Figure 5c,g and Figure 5d,h show saturation and value (brightness) for the simulations (solid circles) and experiments (hollow circles), respectively. Discrepancies are also visible for the same colors. Most of the reflection colors have saturation values close to 1 , whereas some of them decrease in the red range due to the similar reason for the hue; however, they still have good performances in saturation. The decrease of saturation and increase of value observed experimentally can be explained by the same reason as the convergence effect observed previously in the CIE1931 chromaticity space. In the case of transmission, the saturation is relatively low when compared with the reflection, but the brightness maintains a high level-more than 0.8 with some colors reaching even 1 . In Figure 5, the average levels of $\Delta^{\text {Hue }}$, saturation and value, are represented with black dashed lines and numbers, which quantitatively explain the better performances for colors in transmission than in reflection, when considering the agreement between simulated and experimental results in the CIE1931 chromaticity space.

Figure $6 \mathrm{a}, \mathrm{b}$ shows the experimental reflection and transmission colors for fabricated capital letters in serif font Georgia captured by optical microscope, respectively. The parameter combinations for radii and periods are carefully chosen from the previously described 16 cases to generate the rainbow colors in reflection. The colors are kept complementary in reflection and transmission, and the full details of this famous font are clearly reproduced in our experiment. From the SEM image of the middle part of letter "E" in Figure $6 c$, we can find that even a few periods of the nanodisks can maintain a good color performance. This example, with its simulations and experiments, indicates that the structure discussed here could be an appealing candidate for plasmonic color applications. a

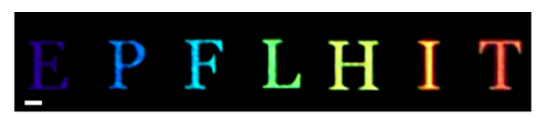

b
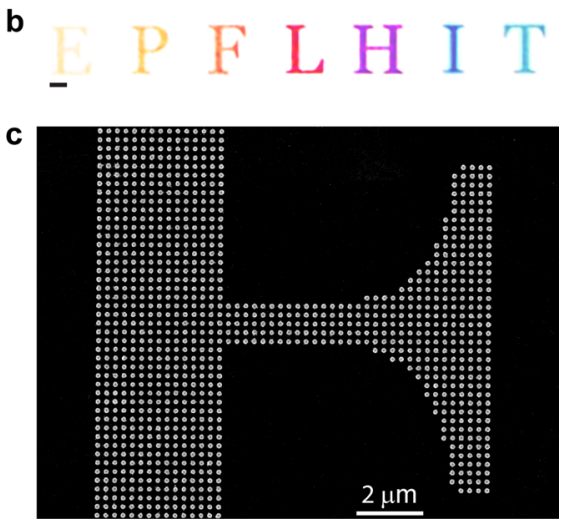

Figure 6. Experimental (a) reflection and (b) transmission colors for fabricated letters in serif font Georgia. Scale bar: $10 \mu \mathrm{m}$. (c) SEM image for the middle part of the letter "E". Scale bar: $2 \mu \mathrm{m}$.

\section{CONCLUSION}

In summary, we have proposed a practical way to realize high saturation and brightness colors by using tandem nanodisks made of $\mathrm{Ag}-\mathrm{Al}_{2} \mathrm{O}_{3}-\mathrm{Ag}$, in which the hybridized mode between the in-phase electric dipole mode and Wood's anomaly dominates the color generation. Since the plasmonic resonances can be tailored by changing the radius of the nanodisk and the period of the array, 16 samples with different parameters have been examined and their reflection and transmission colors analyzed in CIE1931, sRGB, and HSV color spaces. The experimental results agree well with simulations. This tandem structure has many advantages, such as tunability and robustness for full color generation, ease of fabrication due to a relatively large nanodisk radius, even for short wavelength colors, high saturation and brightness, and complementary colors in reflection and transmission directions. We believe that this system could provide a general platform for plasmonic color printing, high fidelity color rendering, spectral filtering, sensors, etc. In addition, dynamic color tunability could also be achieved when the structure is combined with electrochemical bias, ${ }^{81}$ liquid crystal, $^{82}$ or other refractive index tunable materials.

\section{METHODS}

Fabrication. $\mathrm{A} \mathrm{Ag}-\mathrm{Al}_{2} \mathrm{O}_{3}-\mathrm{Ag}$ sandwich film was first deposited on a float glass wafer (thickness of $550 \mu \mathrm{m}$ Guinchard, optical glass Switzerland) using an evaporator (Leybold Optics LAB600H, pressure $1 \times 10^{-5} \mathrm{mbar}$ ) with deposition rates of $8 \AA / \mathrm{s}$ for $\mathrm{Ag}$ and $4 \AA / \mathrm{s}$ for $\mathrm{Al}_{2} \mathrm{O}_{3}$. Then a $20 \mathrm{~nm} \mathrm{Si} \mathrm{sacrificial} \mathrm{layer} \mathrm{was} \mathrm{deposited} \mathrm{on} \mathrm{top} \mathrm{of} \mathrm{the}$ sandwich film by magnetic sputtering (Pfeiffer SPIDER 600) at room temperature with a deposition rate of $11 \AA \AA$ s. Afterward, the wafer was dehydrated for $5 \mathrm{~min}$ at $180{ }^{\circ} \mathrm{C}$ on a hot plate. Subsequently, $180 \mathrm{~nm}$ hydrogen silsesquioxane (HSQ) (XR 1541006 Dow Corning) was spin-coated at $2000 \mathrm{rpm}$. The nanodisks were patterned using electron beam lithography (Vistec EBPG5000 system) with a $100 \mathrm{keV}$ electron beam (current $200 \mathrm{pA}$ ). The patterned wafer was developed with tetramethylammonium hydroxide solution for $30 \mathrm{~s}$, washed with DI water for $10 \mathrm{~min}$, and dried with nitrogen gas. The sample was ionetched at $-10{ }^{\circ} \mathrm{C}$ for $150 \mathrm{~s}$ using an argon ion beam etcher (Veeco Nexus IBE350). Finally, HSQ and Si layers were removed by wet etching at $60{ }^{\circ} \mathrm{C}$ for $1 \mathrm{~min}(\mathrm{KOH}, 40 \%$, Plade Six Sigma), and the sample was showered with DI water before being dried with nitrogen gas. To remove contamination and residues, the sample was immersed 
in deionized water at $60^{\circ} \mathrm{C}$ for $2 \mathrm{~h}$ and dried with nitrogen gas. The sample was stored in a Dry Keeper (Sato Keiryoki MFG Co. Ltd.), which was purged continuously with $\mathrm{Ar}_{2}$ gas at $20{ }^{\circ} \mathrm{C}$ at $1.013 \mathrm{~Pa}$ pressure and $5 \mathrm{~nL} / \mathrm{min}$ to keep the metal nanostructures stable.

SEM and Optical Characterization. The SEM micrographs of the nanodisks were characterized with a Zeiss Merlin field emission scanning electron microscope (Carl Zeiss Jena $\mathrm{GmbH}$ ) at $2 \mathrm{keV}$. Reflection and transmission color images of the sample were characterized using the Nikon Optiphot 200 inspection microscope $(10 \times, N A=0.25$ objective). A CCD camera (Digital Sight DS-2Mv, Nikon) was used to record the images of the sample, and the images were processed with NIS-Elements F3.2 software. The spectra (reflection and transmission) were characterized with a visible spectroscopy system based on an inverted optical microscope (Olympus IX-71) coupled to a spectrometer (Jobin Yvon Horiba Triax 550). The sample was illuminated using a halogen white light source focused onto the sample using an objective $(20 \times, \mathrm{NA}=0.4)$. The reflected light was collected through the same objective and recorded using a spectrometer. The reflected intensity was normalized by the spectrum of the lamp obtained by reflection measurements with a silver mirror (Thorlabs PF 10-03-P01). The transmission spectra were measured with the same objective. The transmitted light through the nanodisk array was normalized by the transmitted light through a reference glass substrate. A polarizer (LPVIS050-MP, Thorlabs) was used to set linear polarized light illumination for both reflection and transmission measurements.

Numerical Simulation. Optimization of the nanodisk thicknesses, parameter sweep of the radius, period of the tandem nanodisk arrays, and calculations of electric and magnetic fields were performed by a FDTD method based commercial software (FDTD Solutions, Lumerical, Canada). The charge distribution was calculated with a SIE based in house code. The permittivity of $\mathrm{Ag}$ was interpolated from Johnson and Christy's data. ${ }^{83}$ The refractive index of $\mathrm{Al}_{2} \mathrm{O}_{3}$ was from the Palik's data, ${ }^{84}$ and the refractive index of the $\mathrm{SiO}_{2}$ substrate was set to 1.52 for all of the calculations.

\section{ASSOCIATED CONTENT}

\section{S Supporting Information}

The Supporting Information is available free of charge on the ACS Publications website at DOI: 10.1021/acsnano.6b08465.

Optimization of the thicknesses of $H$ and $h$ for a sandwich structure; process of fabrication; comparison of sandwich and single nanodisk structures; period and radius combinations for 16 colors; resonance dependence on the incident angle for TE and TM polarizations; charge distribution of high-order modes, magnetic field of out-of-phase mode; reflection and transmission spectra with different radii at fixed array period; period and radius combinations for 16 colors; experimental reflection and transmission spectra for 16 samples; simulated colors; color-matching functions; comparison of data in CIEXYZ, sRGB color spaces; and HSV color cones (PDF)

\section{AUTHOR INFORMATION}

\section{Corresponding Authors}

*E-mail: jingwenz@hit.edu.cn.

*E-mail: olivier.martin@epfl.ch.

ORCID

Hao Wang: 0000-0001-5388-6691

Chen Yan: 0000-0002-3171-0449

Olivier J. F. Martin: 0000-0002-9574-3119

Notes

The authors declare no competing financial interest.

\section{ACKNOWLEDGMENTS}

The authors thank Dr. T.V. Raziman and Mr. Kuang-yu Yang for fruitful discussions. This work is supported by a grant from National Natural Science Foundation of China (NSFC) (No. 11374076).

\section{REFERENCES}

(1) Barchiesi, D. Lycurgus Cup: Inverse Problem Using Photographs for Characterization of Matter. J. Opt. Soc. Am. A 2015, 32, 15441555.

(2) Gu, Y.; Zhang, L.; Yang, J. K.; Yeo, S. P.; Qiu, C.-W. Color Generation via Subwavelength Plasmonic Nanostructures. Nanoscale 2015, 7, 6409-6419.

(3) Hedayati, M. K.; Elbahri, M. Review of Metasurface Plasmonic Structural Color. Plasmonics 2016, 1-17.

(4) Kristensen, A.; Yang, J. K. W.; Bozhevolnyi, S. I.; Link, S.; Nordlander, P.; Halas, N. J.; Mortensen, N. A. Plasmonic Colour Generation. Nat. Rev. Mater. 2016, 2, 16088.

(5) King, N. S.; Liu, L.; Yang, X.; Cerjan, B.; Everitt, H. O.; Nordlander, P.; Halas, N. J. Fano Resonant Aluminum Nanoclusters for Plasmonic Colorimetric Sensing. ACS Nano 2015, 9, 1062810636.

(6) Li, Z.; Wang, W.; Rosenmann, D.; Czaplewski, D. A.; Yang, X.; Gao, J. All-Metal Structural Color Printing Based on Aluminum Plasmonic Metasurfaces. Opt. Express 2016, 24, 20472-20480.

(7) Olson, J.; Manjavacas, A.; Basu, T.; Huang, D.; Schlather, A. E.; Zheng, B.; Halas, N. J.; Nordlander, P.; Link, S. High Chromaticity Aluminum Plasmonic Pixels for Active Liquid Crystal Displays. ACS Nano 2016, 10, 1108-1117.

(8) Olson, J.; Manjavacas, A.; Liu, L.; Chang, W.-S.; Foerster, B.; King, N. S.; Knight, M. W.; Nordlander, P.; Halas, N. J.; Link, S. Vivid, Full-Color Aluminum Plasmonic Pixels. Proc. Natl. Acad. Sci. U. S. A. 2014, 111, 14348-14353.

(9) Shrestha, V. R.; Lee, S.-S.; Kim, E.-S.; Choi, D.-Y. Aluminum Plasmonics Based Highly Transmissive Polarization-Independent Subtractive Color Filters Exploiting a Nanopatch Array. Nano Lett. 2014, 14, 6672-6678.

(10) Wang, L.; Ng, R. J. H.; Safari Dinachali, S.; Jalali, M.; Yu, Y.; Yang, J. K. Large Area Plasmonic Color Palettes with Expanded Gamut Using Colloidal Self-Assembly. ACS Photonics 2016, 3, 627-633.

(11) Si, G.; Zhao, Y.; Lv, J.; Lu, M.; Wang, F.; Liu, H.; Xiang, N.; Huang, T. J.; Danner, A. J.; Teng, J.; et al. Reflective Plasmonic Color Filters Based on Lithographically Patterned Silver Nanorod Arrays. Nanoscale 2013, 5, 6243-6248.

(12) Yu, R.; Mazumder, P.; Borrelli, N. F.; Carrilero, A.; Ghosh, D. S.; Maniyara, R. A.; Baker, D.; García de Abajo, F. J.; Pruneri, V. Structural Coloring of Glass Using Dewetted Nanoparticles and Ultrathin Films of Metals. ACS Photonics 2016, 3, 1194-1201.

(13) Goh, X. M.; Ng, R. J. H.; Wang, S.; Tan, S. J.; Yang, J. K. Comparative Study of Plasmonic Colors from All-Metal Structures of Posts and Pits. ACS Photonics 2016, 3, 1000-1009.

(14) Si, G.; Zhao, Y.; Leong, E. S. P.; Lv, J.; Liu, Y. J. Incident-Angle Dependent Color Tuning from a Single Plasmonic Chip. Nanotechnology 2014, 25, 455203.

(15) Lovera, P.; Jones, D.; Corbett, B.; O’Riordan, A. Polarization Tunable Transmission through Plasmonic Arrays of Elliptical Nanopores. Opt. Express 2012, 20, 25325-25332.

(16) Genet, C.; Ebbesen, T. Light in Tiny Holes. Nature 2007, 445, $39-46$.

(17) Yokogawa, S.; Burgos, S. P.; Atwater, H. A. Plasmonic Color Filters for CMOS Image Sensor Applications. Nano Lett. 2012, 12, 4349-4354.

(18) Sun, L.; Hu, X.; Zeng, B.; Wang, L.; Yang, S.; Tai, R.; Fecht, H.; Zhang, D.; Jiang, J. Effect of Relative Nanohole Position on Colour Purity of Ultrathin Plasmonic Subtractive Colour Filters. Nanotechnology 2015, 26, 305204.

(19) Cheng, F.; Yang, X.; Rosenmann, D.; Stan, L.; Czaplewski, D.; Gao, J. Enhanced Structural Color Generation in Aluminum 
Metamaterials Coated with a Thin Polymer Layer. Opt. Express 2015, 23, 25329-25339.

(20) Inoue, D.; Miura, A.; Nomura, T.; Fujikawa, H.; Sato, K.; Ikeda, N.; Tsuya, D.; Sugimoto, Y.; Koide, Y. Polarization Independent Visible Color Filter Comprising an Aluminum Film with SurfacePlasmon Enhanced Transmission through a Subwavelength Array of Holes. Appl. Phys. Lett. 2011, 98, 093113.

(21) Kumar, K.; Duan, H.; Hegde, R. S.; Koh, S. C.; Wei, J. N.; Yang, J. K. Printing Colour at the Optical Diffraction Limit. Nat. Nanotechnol. 2012, 7, 557-561.

(22) Clausen, J. S.; Højlund-Nielsen, E.; Christiansen, A. B.; Yazdi, S.; Grajower, M.; Taha, H.; Levy, U.; Kristensen, A.; Mortensen, N. A. Plasmonic Metasurfaces for Coloration of Plastic Consumer Products. Nano Lett. 2014, 14, 4499-4504.

(23) James, T. D.; Mulvaney, P.; Roberts, A. The Plasmonic Pixel: Large Area, Wide Gamut Color Reproduction Using Aluminum Nanostructures. Nano Lett. 2016, 16, 3817-3823.

(24) Lu, B.-R.; Xu, C.; Liao, J.; Liu, J.; Chen, Y. High-Resolution Plasmonic Structural Colors from Nanohole Arrays with Bottom Metal Disks. Opt. Lett. 2016, 41, 1400-1403.

(25) Zhu, X.; Vannahme, C.; Højlund-Nielsen, E.; Mortensen, N. A.; Kristensen, A. Plasmonic Colour Laser Printing. Nat. Nanotechnol. 2015, 11, 325-329.

(26) Tan, S. J.; Zhang, L.; Zhu, D.; Goh, X. M.; Wang, Y. M.; Kumar, K.; Qiu, C.-W.; Yang, J. K. Plasmonic Color Palettes for Photorealistic Printing with Aluminum Nanostructures. Nano Lett. 2014, 14, 40234029.

(27) Miyata, M.; Hatada, H.; Takahara, J. Full-Color Subwavelength Printing with Gap-Plasmonic Optical Antennas. Nano Lett. 2016, 16, 3166-3172.

(28) Goh, X. M.; Zheng, Y.; Tan, S. J.; Zhang, L.; Kumar, K.; Qiu, C.W.; Yang, J. K. Three-Dimensional Plasmonic Stereoscopic Prints in Full Colour. Nat. Commun. 2014, 5, 5361.

(29) Ellenbogen, T.; Seo, K.; Crozier, K. B. Chromatic Plasmonic Polarizers for Active Visible Color Filtering and Polarimetry. Nano Lett. 2012, 12, 1026-1031.

(30) Li, Z.; Clark, A. W.; Cooper, J. M. Dual Color Plasmonic Pixels Create a Polarization Controlled Nano Color Palette. ACS Nano 2016, 10, 492-498.

(31) Lin, Y.; Chen, Z.; Shen, C. The Simulation and Fabrication of $\mathrm{Ag} / \mathrm{SiO}_{2} / \mathrm{Ag}$ Thin Films Color Filter. Phys. Procedia 2012, 32, 19-30.

(32) Li, Z.; Butun, S.; Aydin, K. Large-Area, Lithography-Free Super Absorbers and Color Filters at Visible Frequencies Using Ultrathin Metallic Films. ACS Photonics 2015, 2, 183-188.

(33) Park, C.-S.; Shrestha, V. R.; Lee, S.-S.; Choi, D.-Y. TransReflective Color Filters Based on a Phase Compensated Etalon Enabling Adjustable Color Saturation. Sci. Rep. 2016, 6, 25496.

(34) Yang, Z.; Zhou, Y.; Chen, Y.; Wang, Y.; Dai, P.; Zhang, Z.; Duan, H. Reflective Color Filters and Monolithic Color Printing Based on Asymmetric Fabry-Perot Cavities Using Nickel as a Broadband Absorber. Adv. Opt. Mater. 2016, 4, 1196-1202.

(35) Mao, K.; Shen, W.; Yang, C.; Fang, X.; Yuan, W.; Zhang, Y.; Liu, $\mathrm{X}$. Angle Insensitive Color Filters in Transmission Covering the Visible Region. Sci. Rep. 2016, 6, 19289.

(36) Yang, C.; Shen, W.; Zhang, Y.; Li, K.; Fang, X.; Zhang, X.; Liu, $\mathrm{X}$. Compact Multilayer Film Structure for Angle Insensitive Color Filtering. Sci. Rep. 2015, 5, 9285.

(37) Lee, K. T.; Seo, S.; Guo, L. J. High-Color-Purity Subtractive Color Filters with a Wide Viewing Angle Based on Plasmonic Perfect Absorbers. Adv. Opt. Mater. 2015, 3, 347-352.

(38) Yang, C.; Shen, W.; Zhang, Y.; Ye, Z.; Zhang, X.; Li, K.; Fang, $\mathrm{X}$.; Liu, X. Color-Tuning Method by Filling Porous Alumina Membrane Using Atomic Layer Deposition Based on MetalDielectric-Metal Structure. Appl. Opt. 2014, 53, A142-A147.

(39) Shrestha, V. R.; Lee, S.-S.; Kim, E.-S.; Choi, D.-Y. NonIridescent Transmissive Structural Color Filter Featuring Highly Efficient Transmission and High Excitation Purity. Sci. Rep. 2014, 4, 4921.
(40) Xue, J.; Zhou, Z.-K.; Wei, Z.; Su, R.; Lai, J.; Li, J.; Li, C.; Zhang, T.; Wang, X.-H. Scalable, Full-Colour and Controllable Chromotropic Plasmonic Printing. Nat. Commun. 2015, 6, 8906.

(41) Uddin, M. J.; Magnusson, R. Efficient Guided-Mode-Resonant Tunable Color Filters. IEEE Photonics Technol. Lett. 2012, 24, 15521554.

(42) Xu, T.; Wu, Y.-K.; Luo, X.; Guo, L. J. Plasmonic Nanoresonators for High-Resolution Colour Filtering and Spectral Imaging. Nat. Commun. 2010, 1, 59.

(43) Lochbihler, H. Reflective Colored Image Based on MetalDielectric-Metal-Coated Gratings. Opt. Lett. 2013, 38, 1398-1400.

(44) Kaplan, A. F.; Xu, T.; Wu, Y.-K.; Guo, L. J. Multilayer Pattern Transfer for Plasmonic Color Filter Applications. J. Vac. Sci. Technol., B: Nanotechnol. Microelectron.: Mater., Process., Meas., Phenom. 2010, 28, $\mathrm{C} 6 \mathrm{O} 60-\mathrm{C} 6 \mathrm{O} 63$.

(45) Yu, C.-J.; Liang, Y.-P.; Hong, H.-F.; Wang, C.-M. Transmissive Color Filtering Using Plasmonic Multilayer Structure. Opt. Eng. (Bellingham, WA, U. S.) 2012, 51, 044001.

(46) Cai, W.; Chettiar, U. K.; Yuan, H.-K.; de Silva, V. C.; Kildishev, A. V.; Drachev, V. P.; Shalaev, V. M. Metamagnetics with Rainbow Colors. Opt. Express 2007, 15, 3333-3341.

(47) Shrestha, V. R.; Lee, S.-S.; Kim, E.-S.; Choi, D.-Y. PolarizationTuned Dynamic Color Filters Incorporating a Dielectric-Loaded Aluminum Nanowire Array. Sci. Rep. 2015, 5, 12450.

(48) Zheng, J.; Ye, Z.-C.; Sheng, Z.-M. Reflective Low-Sideband Plasmonic Structural Colors. Opt. Mater. Express 2016, 6, 381-387.

(49) Lütolf, F.; Stalder, M.; Martin, O. J. Metallized Gratings Enable Color Effects and Floating Screen Films by First-Order Diffraction. Adv. Opt. Mater. 2015, 3, 1793-1799.

(50) Duempelmann, L.; Luu-Dinh, A.; Gallinet, B.; Novotny, L. Four-Fold Color Filter Based on Plasmonic Phase Retarder. ACS Photonics 2016, 3, 190-196.

(51) Shen, Y.; Rinnerbauer, V.; Wang, I.; Stelmakh, V.; Joannopoulos, J. D.; Soljačíc, M. Structural Colors from Fano Resonances. ACS Photonics 2015, 2, 27-32.

(52) Yang, S.-C.; Richter, K.; Fischer, W.-J. Multicolor Generation Using Silicon Nanodisk Absorber. Appl. Phys. Lett. 2015, 106, 081112.

(53) Proust, J.; Bedu, F.; Gallas, B.; Ozerov, I.; Bonod, N. AllDielectric Colored Metasurfaces with Silicon Mie Resonators. ACS Nano 2016, 10, 7761-7767.

(54) Westland, S.; Ripamonti, C. Computational Colour Science Using MATLAB; John Wiley \& Sons: Chichester, U.K., 2004.

(55) Pakizeh, T.; Dmitriev, A.; Abrishamian, M.; Granpayeh, N.; Käll, M. Structural Asymmetry and Induced Optical Magnetism in Plasmonic Nanosandwiches. J. Opt. Soc. Am. B 2008, 25, 659-667.

(56) Pakizeh, T.; Abrishamian, M.; Granpayeh, N.; Dmitriev, A.; Käll, M. Magnetic-Field Enhancement in Gold Nanosandwiches. Opt. Express 2006, 14, 8240-8246.

(57) Mendoza-Galvan, A.; Järrendahl, K.; Dmitriev, A.; Pakizeh, T.; Käll, M.; Arwin, H. Optical Response of Supported Gold Nanodisks. Opt. Express 2011, 19, 12093-12107.

(58) Halas, N. J.; Lal, S.; Chang, W.-S.; Link, S.; Nordlander, P. Plasmons in Strongly Coupled Metallic Nanostructures. Chem. Rev. 2011, 111, 3913-3961.

(59) Su, K.; Wei, Q.; Zhang, X. Tunable and Augmented Plasmon Resonances of $\mathrm{Au} / \mathrm{SiO}_{2} / \mathrm{Au}$ Nanodisks. Appl. Phys. Lett. 2006, 88, 063118 .

(60) Su, K.-H.; Durant, S.; Steele, J. M.; Xiong, Y.; Sun, C.; Zhang, X. Raman Enhancement Factor of a Single Tunable Nanoplasmonic Resonator. J. Phys. Chem. B 2006, 110, 3964-3968.

(61) Strohfeldt, N.; Zhao, J.; Tittl, A.; Giessen, H. Sensitivity Engineering in Direct Contact Palladium-Gold Nano-Sandwich Hydrogen Sensors [Invited]. Opt. Mater. Express 2015, 5, 2525-2535.

(62) Sun, C.; Su, K.-H.; Valentine, J.; Rosa-Bauza, Y. T.; Ellman, J. A.; Elboudwarej, O.; Mukherjee, B.; Craik, C. S.; Shuman, M. A.; Chen, F. F.; et al. Time-Resolved Single-Step Protease Activity Quantification Using Nanoplasmonic Resonator Sensors. ACS Nano 2010, 4, 978984. 
(63) Kuttge, M.; García de Abajo, F. J.; Polman, A. Ultrasmall Mode Volume Plasmonic Nanodisk Resonators. Nano Lett. 2010, 10, 15371541.

(64) Tserkezis, C.; Papanikolaou, N.; Gantzounis, G.; Stefanou, N. Understanding Artificial Optical Magnetism of Periodic MetalDielectric-Metal Layered Structures. Phys. Rev. B: Condens. Matter Mater. Phys. 2008, 78, 165114.

(65) Ekinci, Y.; Christ, A.; Agio, M.; Martin, O.; Solak, H.; Löffler, J. Electric and Magnetic Resonances in Arrays of Coupled Gold Nanoparticle in-Tandem Pairs. Opt. Express 2008, 16, 13287-13295.

(66) Mendoza-Galván, A.; Järrendahl, K.; Dmitriev, A.; Pakizeh, T.; Käll, M.; Arwin, H. Fano Interference in Supported Gold Nanosandwiches with Weakly Coupled Nanodisks. Opt. Express 2012, 20, 29646-29658.

(67) Jeyaram, Y.; Jha, S. K.; Agio, M.; Löffler, J. F.; Ekinci, Y. Magnetic Metamaterials in the Blue Range Using Aluminum Nanostructures. Opt. Lett. 2010, 35, 1656-1658.

(68) Qiu, L.; Wang, S.; Liu, H.; Li, T.; Zhu, S.; Zhang, X. Magnetic Plasmon in Coupled Nanosandwich Structure. J. Opt. Soc. Am. B 2011, $28,1655-1660$.

(69) Dmitriev, A.; Pakizeh, T.; Käll, M.; Sutherland, D. S. Gold-SilicaGold Nanosandwiches: Tunable Bimodal Plasmonic Resonators. Small 2007, 3, 294-299.

(70) Chang, Y.-C.; Wang, S.-M.; Chung, H.-C.; Tseng, C.-B.; Chang, S.-H. Observation of Absorption-Dominated Bonding Dark Plasmon Mode from Metal-Insulator-Metal Nanodisk Arrays Fabricated by Nanospherical-Lens Lithography. ACS Nano 2012, 6, 3390-3396.

(71) Frederiksen, M.; Bochenkov, V. E.; Ogaki, R.; Sutherland, D. S. Onset of Bonding Plasmon Hybridization Preceded by Gap Modes in Dielectric Splitting of Metal Disks. Nano Lett. 2013, 13, 6033-6039.

(72) Thyagarajan, K.; Santschi, C.; Langlet, P.; Martin, O. J. Highly Improved Fabrication of $\mathrm{Ag}$ and $\mathrm{Al}$ Nanostructures for $\mathrm{UV}$ and Nonlinear Plasmonics. Adv. Opt. Mater. 2016, 4, 871-876.

(73) Lumerical FDTD Solutions; http://www.lumerical.com (accessed Dec 1, 2016).

(74) Gallinet, B.; Kern, A. M.; Martin, O. J. Accurate and Versatile Modeling of Electromagnetic Scattering on Periodic Nanostructures with a Surface Integral Approach. J. Opt. Soc. Am. A 2010, 27, 22612271.

(75) Gallinet, B.; Martin, O. J. Influence of Electromagnetic Interactions on the Line Shape of Plasmonic Fano Resonances. ACS Nano 2011, 5, 8999-9008.

(76) Auguié, B.; Barnes, W. L. Collective Resonances in Gold Nanoparticle Arrays. Phys. Rev. Lett. 2008, 101, 143902.

(77) Schmidt, F.-P.; Ditlbacher, H.; Hohenester, U.; Hohenau, A.; Hofer, F.; Krenn, J. R. Dark Plasmonic Breathing Modes in Silver Nanodisks. Nano Lett. 2012, 12, 5780-5783.

(78) Krug, M. K.; Reisecker, M.; Hohenau, A.; Ditlbacher, H.; Trügler, A.; Hohenester, U.; Krenn, J. R. Probing Plasmonic Breathing Modes Optically. Appl. Phys. Lett. 2014, 105, 171103.

(79) ASTM. E308-01, Standard Practice for Computing the Colors of Objects by Using the CIE System, 2001; pp 1-32.

(80) Wyman, C.; Sloan, P.-P.; Shirley, P. Simple Analytic Approximations to the CIE XYZ Color Matching Functions. J. Comput. Graphics Technol. 2013, 2, 1-11.

(81) Wang, G.; Chen, X.; Liu, S.; Wong, C.; Chu, S. Mechanical Chameleon through Dynamic Real-Time Plasmonic Tuning. ACS Nano 2016, 10, 1788-1794.

(82) Franklin, D.; Chen, Y.; Vazquez-Guardado, A.; Modak, S.; Boroumand, J.; Xu, D.; Wu, S.-T.; Chanda, D. PolarizationIndependent Actively Tunable Colour Generation on Imprinted Plasmonic Surfaces. Nat. Commun. 2015, 6, 7337.

(83) Johnson, P. B.; Christy, R.-W. Optical Constants of the Noble Metals. Phys. Rev. B 1972, 6, 4370.

(84) Palik, E. D. Handbook of Optical Constants of Solids; Academic Press: San Diego, CA, 1998; Vol. 3. 